\title{
Summen aufeinander folgender Quadrate, die ein Quadrat ergeben
}

\author{
Josef Rung und Johann Werner ${ }^{\dagger}$ \\ Josef Rung, Jahrgang 1953, studierte Mathematik und Physik. Er bildet am Hans-Lein- \\ berger-Gymnasium in Landshut angehende Mathematiklehrer aus. Neben Veröffentli- \\ chungen zur Algebra und algebraischen Geometrie hat er auch ein Buch über sphäri- \\ sche Trigonometrie und eine „Zahlentheorie für Einsteiger“ mitverfasst. \\ Johann Werner wurde 1923 geboren. Nach dem Studium der Mathematik und Physik \\ war er lange Zeit als Mathematik- und Physiklehrer tätig, viele Jahre auch in Grie- \\ chenland. Danach leitete er bis zu seiner Pensionierung im Jahre 1988 das Clavius- \\ Gymnasium in Bamberg.
}

\section{Einleitung}

E. Lucas hat im 19. Jahrhundert das folgende Problem gestellt (vgl. [2], [3]): „Für welche $n \in \mathbb{N}$ hat die Gleichung

(L)

$$
\sum_{j=0}^{n-1}(x+j)^{2}=y^{2}
$$

Lösungen $(x, y)$ über $\mathbb{N}$ ?“

$\dagger$ Herr Johann Werner ist am 12. November 2004 verstorben.

Summen aufeinander folgender Quadratzahlen haben neuerdings verstärkt Interesse gefunden. Beispielsweise hat Warlimont 1998 im Journal of Number Theory (Vol. 68, pp. 87-98) etwa die Frage untersucht, wie viele natürliche Zahlen (unterhalb einer gegebenen Schranke) Summen aufeinander folgender Quadrate sind. Bremner u.a. studierten im selben Journal (Vol. 62, pp. 39-70), für welche „Startquadrate“ solche Summen auf Quadratzahlen führen. Die Autoren stellen sich hier der alten Aufgabe, für welche Summandenzahlen derartige Quadratsummen wieder Quadrate ergeben können. Sie geben polynomiale Scharen solcher Zahlen an und lösen für rationale Summanden die Aufgabe vollständig. In allen drei Themenkreisen gibt es noch ungelöste Probleme. 
Die vorliegende Arbeit verfolgt folgende Ziele:

1. Wir geben Polynome $p(k)$ derart an, dass für $n=p(k), k \in \mathbb{N}$, Lösungen von (L) mit $x, y \in \mathbb{N}$ existieren.

2. Wir beweisen notwendige und hinreichende Bedingungen dafür, dass (L) rationale Lösungen $x, y$ besitzt.

3. Wir leiten notwendige (aber nicht hinreichende) Bedingungen her für die Existenz natürlichzahliger Lösungen.

Dazu wird (L) in eine etwas andere Gestalt gebracht: Führt man die Summierungen aus, multipliziert mit 4 und setzt $2 y=: z$ und $2 x+(n-1)=: u$, so erhält man für (L) die äquivalenten Formulierungen

bzw.

$$
n u^{2}+\frac{n(n-1)(n+1)}{3}=z^{2}
$$

$$
3 n u^{2}+n(n-1)(n+1)=3 z^{2} \text {. }
$$

Dies gilt unter den Nebenbedingungen:

(i) $z \equiv 0(\bmod 2)$ und

(ii) $u \equiv 1(\bmod 2)$ genau wenn $n \equiv 0(\bmod 2)$

Da für nichtquadratische $n$ mit jeder ganzzahligen Lösung $u$ eine größere existiert, kann daraus stets ein positives $x$ bestimmt werden. Es muss $z$ gerade und $u$ genau dann ungerade sein, wenn $n$ gerade ist.

\section{Polynomiale $n$, die natürlichzahlige Lösungen zulassen}

Satz 1 Ganzzahlige positive Lösungen existieren für alle

a) $n=3 k^{2}-1, k \in \mathbb{N}$,

b) $n=(6 k \pm 1)^{2}, n \geq 49, k \in \mathbb{N}$.

Hat (L) wenigstens eine ganzzahlige Lösung und ist $n$ keine Quadratzahl, dann hat (L) unendlich viele ganzzahlige Lösungen.

Beweis. a) Für $n=3 k^{2}-1, u=k \in \mathbb{N}$ und $z=k \cdot\left(3 k^{2}-1\right)$ ist (1) erfüllt, $z$ gerade und $u$ genau dann ungerade, wenn $n$ gerade ist. Das beweist a). Die Existenz unendlich vieler ganzzahliger Lösungen folgt etwa aus [5, Theorem 104].

b) Für $n=(6 k \pm 1)^{2}, n \geq 49, u=\frac{n^{2}-49}{24}$ und $z=(6 k \pm 1)(u+4)>0$ ist (1) erfüllt. $z$ und $u=\left(9 k^{2} \pm 3 k+2\right)\left(6 k^{2} \pm 2 k-1\right)$ sind gerade. Falls $n>26$, ist $u>n-1$, also $x>0$. Das beweist $b)$.

Für $n=25$ findet man $\sum_{j=0}^{24}(0+j)^{2}=70^{2}$, und man überlegt sich leicht, dass dies die einzige Summe von 25 aufeinander folgenden Quadratzahlen ist, die wieder ein Quadrat ergibt. 


\section{Rationale Lösungen für Lucas' Gleichungen}

Für eine natürliche Zahl $n$ und eine Primzahl $p$ bezeichne $v_{p}(n)$ den Exponenten von $p$ in der Primfaktorzerlegung von $n .\left(\frac{n}{p}\right)$ ist das Legendre-Symbol.

Satz $2(\mathrm{~L})$ ist über $\mathbb{Q}$ genau dann lösbar, wenn für alle Primzahlen $p>3$ die folgenden Bedingungen erfüllt sind:

1) $\left(\frac{3}{p}\right)^{v_{p}(n)}=1$ und

2) $\left(\frac{-1}{p}\right)^{v_{p}\left(n^{\prime}\right)}=1$, wobei $n^{\prime}= \begin{cases}n+1 & \text { falls } n+1 \not \equiv 0(\bmod 3), \\ \frac{n+1}{3} & \text { falls } n+1 \equiv 0(\bmod 3) .\end{cases}$

Falls 3 ein Teiler von $n$ ist, kommt noch die folgende Bedingung hinzu:

3) $\frac{n}{3^{v_{3}(n)}} \equiv(-1)^{v_{3}(n)}(\bmod 3)$.

Beweis. Die Gleichungen (L) und (1) sind über $\mathbb{Q}$ äquivalent, denn sie sind unter den Nebenbedingungen (i), (ii) schon über $\mathbb{N}$ äquivalent. Notwendig und hinreichend für die Existenz rationaler Lösungen von (1) ist offensichtlich die Existenz rationaler Lösungen $(U, V, Z)$ von

$$
Z^{2}-n U^{2}-\frac{n(n-1)(n+1)}{3} V^{2}=0 .
$$

Um Letzteres zu begründen muss nur gezeigt werden, dass auch im Falle einer rationalen Lösung $(U, V, Z)$ von (3) mit $V=0$ eine rationale Lösung von (1) existiert. Falls nun $V=0$ ist, dann ist $n=k^{2}$ eine Quadratzahl. Mit $N:=\frac{1}{3} n(n-1)(n+1)$ lösen die rationalen $u:=\frac{N-1}{2 k}$ und $z:=\frac{N+1}{2}$ die Gleichung (1).

Es genügt also zu zeigen, dass die in Satz 2 genannten Bedingungen notwendig und hinreichend für die Lösbarkeit von (3) sind. Dazu verwenden wir das Hasse-Prinzip und benutzen die Standardresultate und Bezeichnungen über $p$-adische Zahlen, wie sie (etwa) in [6, S. 19ff.] zu finden sind, insbesondere das Hilbertsymbol $(a, b)=(a, b)_{p}$ im Körper $\mathbb{Q}_{p}$. Von Bedeutung sind vor allem Theorem 1, S. 20, Theorem 3, S. 23 (Hilberts Produktformel) und Theorem 8, S. 41 (Hasse-Minkowski).

Wegen der Vorzeichen der Koeffizienten besitzt Gleichung (3) sicher reelle Lösungen $(U, V, Z) \neq(0,0,0)$. Notwendig für die rationale Lösbarkeit von (3) ist, dass für alle ungeraden Primzahlen $p$ gilt:

$$
H_{p}:=\left(\frac{1}{3} n(n-1)(n+1), n\right)_{p}=1 .
$$

Ist andererseits $H_{p}=1$ für alle ungeraden $p$, dann ist nach der Hilbertschen Produktformel auch $H_{2}=1$. Daher ist , $H_{p}=1$ für alle ungeraden $p$ “ nach Hasse-Minkowski auch hinreichend für die Lösbarkeit von Gleichung (3) (d.h. von (1)) über den rationalen Zahlen.

Wir schreiben künftig, wie oben

$$
N:=\frac{1}{3} n(n-1)(n+1)
$$

und kürzen eine (ungerade) Primzahl stets mit $p$ ab. 
Wir zeigen jetzt, dass die Bedingung (3) äquivalent ist zu den in Satz 2 genannten Bedingungen; dazu sind mehrere Fälle zu unterscheiden:

1. Fall: $n=3 k+1, k \in \mathbb{N}$.

a) Ist $p$ ein Teiler von $n$, also $v_{p}(n)>0$ (und damit $p>3$ ), dann gilt $v_{p}(N)=v_{p}(n)=: a$, $N=k(3 k+1)(3 k+2)=: p^{a} u, n=: p^{a} v$, mit $p$-adischen Einheiten $u, v \in \mathbb{Z}$. Daraus folgt $u=v k(3 k+2)$. Damit ist dann

$$
\begin{gathered}
H_{p}=\left(\frac{-1}{p}\right)^{a^{2}} \cdot\left(\frac{v k(3 k+2)}{p}\right)^{a} \cdot\left(\frac{v}{p}\right)^{a} \\
3 k+2 \equiv 1(\bmod p)\left(\frac{-1}{p}\right)^{a} \cdot\left(\frac{k}{p}\right)^{a} \\
3 k \equiv-1(\bmod p)\left(\frac{3 k}{p}\right)^{a} \cdot\left(\frac{k}{p}\right)^{a}=\left(\frac{3}{p}\right)^{v_{p}(n)} .
\end{gathered}
$$

b) Ist $p$ Teiler von $n-1$ dann rechnet man analog nach: $H_{p}=\left(\frac{n}{p}\right)^{v_{p}(N)}=1$.

c) Ist $p$ Teiler von $n+1$, so ergibt sich: $H_{p}=\left(\frac{3 k+1}{p}\right)^{v_{p}(N)}=\left(\frac{-1}{p}\right)^{v_{p}(n+1)}$.

2. Fall: $n=3 k-1, k \in \mathbb{N}$.

$\overline{\text { a) Ist } p}$ ein Teiler von $n$, also wieder $v_{p}(N)=v_{p}(n)=: a$, ferner $N=k(3 k-1)(3 k-2)=$ $p^{a} u$ und $n=p^{a} v$ mit $p$-adischen Einheiten $u, v$, dann ist $u=v k(3 k-2)$ und man rechnet wie im Fall 1a) aus: $H_{p}=\left(\frac{3}{p}\right)^{v_{p}(n)}$.

b) Wenn $p$ Teiler von $n-1$ ist, dann ergibt sich wieder wie oben: $H_{p}=1$.

c) Ist $p$ schließlich Teiler von $k=\frac{n+1}{3}$, dann ergibt sich mit $v_{p}(n)=0$ :

$$
H_{p}=\left(\frac{-1}{p}\right)^{v_{p}\left(\frac{n+1}{3}\right)} \text {. }
$$

3. Fall: $n=3 k, k \in \mathbb{N}$.

Wenn $p>3$, so unterscheidet man wieder die Fälle

a) $p$ teilt $k$,

b) $p$ teilt $3 k-1$ bzw.

c) $p$ teilt $3 k+1$,

die (wie im 1. Fall) auf
a) $H_{p}=\left(\frac{3}{p}\right)^{v_{p}(n)}$,
b) $H_{p}=1$ bzw.
c) $H_{p}=\left(\frac{-1}{p}\right)^{v_{p}(n+1)}$

führen, also auf das im Satz 2 unter 1) und 2) Genannte.

Es bleibt (für $n=3 k$ ) der Fall $p=3$. Dann ist $N=k(3 k-1)(3 k+1), v_{3}(N)=v_{3}(n)-1$. Mit $v:=\frac{n}{3^{\nu_{3}(n)}}$ ergibt sich $H_{3}=\left(\frac{v}{3}\right)^{\nu_{3}(N)} \cdot\left(\frac{(3 k-1)(3 k+1)}{3}\right)^{\nu_{3}(n)}=(-1)^{\nu_{3}(n)} \cdot\left(\frac{v}{3}\right)^{\nu_{3}(n)-1} \cdot H_{3}$ ist genau dann 1 , wenn $v \equiv(-1)^{\nu_{3}(n)}(\bmod 3)$. Das ergibt schließlich die Bedingung 3$)$ unseres Satzes.

Insgesamt ist nunmehr Satz 2 bewiesen. 


\section{Natürlichzahlige Lösungen von (L)}

Wir formulieren notwendige Bedingungen für die Lösbarkeit von (L) über $\mathbb{N}$ (siehe auch [1]; [4, insbes. 3.2]).

Korollar Eine Lösung der Lucas'schen Gleichung in natürlichen Zahlen ist unmöglich für Summandenzahlen $n$ mit einer der folgenden Eigenschaften:

1) $n$ enthält einen Primfaktor $p \equiv 5$ oder $7(\bmod 12)$ in ungerader als höchster Potenz.

2) $n+1$ enthält 3 in gerader Potenz oder einen Primfaktor $p>3$ mit $p \equiv 3(\bmod 4)$ in ungerader als höchster Potenz.

3) a) $n$ enthält 3 in gerader als höchster Potenz.

b) $n$ enthält 3 in ungerader als höchster Potenz, aber der Restfaktor ist $\equiv 1(\bmod 3)$.

4) $n$ enthält 2 in gerader als höchster Potenz.

Beweis. 1), 2), und 3.b) sind Umformulierungen von Satz 2.

$\mathrm{Zu}$ 4): Für $n=2^{2 s} \cdot r,(r, 2)=1$, ergibt sich aus der in der Einleitung hergeleiteten Gleichung (2):

$$
3 r u^{2}+r\left(n^{2}-1\right)=3 \bar{z}^{2} .
$$

Diese Gleichung ist modulo 4 unerfüllbar: Beachtet man, dass (alles mod 4)

$$
u^{2} \equiv 1, r \equiv 1 \text { oder } 3, \text { und } n^{2}-1 \equiv 3
$$

dann hat die linke Seite den Viererrest 2, während der der rechten Seite 0 oder 3 ist.

Zu 3.a): Aus (2) folgert man für $n=3^{2 s} \cdot r,(r, 3)=1$, die modulo 3 unerfüllbare Gleichung

$$
3 r u^{2}+r\left(n^{2}-1\right)=3 \bar{z}^{2} .
$$

Insgesamt ist nun das Korollar bewiesen.

Die im Korollar formulierten Bedingungen an $n$ für die Lösbarkeit von (1) sind i.Allg. nicht hinreichend, wie die Beispiele $n=842$ (siehe auch [1]) oder 2306 oder 88187 zeigen. Mit Satz 2 ergibt sich leicht, dass in diesen Fällen (L) eine rationale Lösung hat. Mit [5, Theorem 108, S. 205]) kann man durch Rechnung aber definitiv ausschließen, dass positive ganzzahlige Lösungen existieren. (Hinreichende Bedingungen erhält man allerdings, wenn für die zu der binären quadratischen Form (1) gehörenden Diskriminanten die Geschlechter einklassig sind; dies soll aber hier nicht weiter verfolgt werden.) 


\section{Literatur}

[1] Beeckmans, L.: Squares Expressible as Sum of Consecutive Squares. AMM 104 (1994).

[2] Dickson, L.E.: History of the Theory of Numbers, Vol II. New York 1939.

[3] Guy, R.K.: Unsolved Problems in Number Theory. $2^{\text {nd }}$ Ed., New York 1994.

[4] Meurer, A.: Einige Summen natürlicher Zahlen, die ein Quadrat ergeben. Diplomarbeit, Universität Mainz 1995.

[5] Nagell, T.: Introduction to Number Theory. Chelsea, New York 1964.

[6] Serre, J.-P.: A Course in Arithmetic. New York 1973.

Wir danken Herrn Wolfgang Guggenberger für die Erstellung des LaTEX-Files.

\section{Josef Rung}

Katharina-Geisler-Str. 23

D-85356 Freising, Deutschland

e-mail: Josef .Rung@gmx.de 